\title{
MODEL CONSTRUCTION AND EXPERIMENTAL VERIFICATION OF THE EQUIVALENT ELASTIC MODULUS OF A DOUBLE-HELIX WIRE ROPE
}

\author{
Hong Yue Chen, Kun Zhang, Yang Xi Bai \\ School of Mechanical Engineering, Liaoning Technical University, Fuxin, China; e-mail: zhangkunliaoning@163.com \\ YING MA \\ Coal Mining and Designing Department, Tiandi Science and Technology Co., Ltd., Beijing, China
}

HAN Zhong Deng

College of Materials Science and Engineering, Liaoning Technical University, Fuxin, China

\begin{abstract}
To accurately describe mechanical properties of a complex wire rope, a double-helix wire rope is used as an example in this study. According to the spatial structure characteristics of the central helical line of each wire rope, the spatial configuration curve for the double-helix wire rope is obtained by using differential geometry theory. On the basis of this curve, the mathematical model of the equivalent elastic modulus of the wire rope is developed, and the elastic modulus of a $6 \times 7+$ IWS wire rope is measured using a universal tensile testing machine. The experimental results are compared with the predicted results to verify correctness of the elastic modulus prediction of the double-helix wire rope.
\end{abstract}

Keywords: mechanical properties, wire rope, double-helix wire rope, spatial configuration curve, equivalent elastic modulus

\section{Introduction}

Wire ropes are widely used in material-handling machinery because they possess high strength, are light in weight and provide stable and reliable operation. Their safety and reliability directly affect production efficiency and personnel safety. Since the 1950s, numerous domestic and foreign scholars have conducted research on wire ropes. However, because of the complex spiral structure of a wire rope, the theoretical basis required for accurate prediction of its mechanical performance is not yet completely established. Some existing research studies related to this topic are as follows. Stanova et al. (2011) fully considered the spatial spiral structure of a single wire and stranded ropes and developed a parametric mathematical model of the wire rope. Ma et al. (2015) deduced a function expression for the central line of a wire rope based on the Serret-Frenet frame theory. Using the differential geometry theory as the theoretical basis, Hobbs and Nabijou (1995) and Nabijou and Hobs (1995) provided a path expression for a wire on a rope sheave based on the mathematical model of a vertical wire rope. Wu and Cao (2016) deduced the equivalent elastic modulus of a wire rope based on the theory of slender elastic rods. Prawoto and Mazlan (2012) studied mechanical properties of a steel wire rope under tensile load by numerical simulation and experimental methods and obtained the microstructure of fracture in the steel wire rope. Stanová (2013) derived mathematical models of oval strand ropes and used Pro/E software to build geometric models of the ropes. Sathikh et al. (1996) used the Wempner-Ramsey theory to study the asymmetry of the stiffness matrix in a wire-rope elasticity model under bending and torsional loads and validated the model experimentally. Erdönmez and Erdem Imrak (2009) developed a three-dimensional structural model of a double-helix wire rope that took into account friction and slip between the rope cores. Machida and Durelli (1973) defined an expression for the axial force, bending moment and torsional moment in a helix. Elata et al. (2004) proposed a new model 
for simulating the mechanical response of wire ropes with individual steel cord cores. The model fullly considered the double-helix structure of a single wire in the wound strand and provided the stress in the wire layer to estimate global characteristics of the wire rope. Hu et al. (2016) derived initial parameters of different general wire-rope models and developed IWRC6/36WS wire-rope model using MATLAB and Pro/E. The elastic properties of the wire rope under axial tension were analyzed by Abaqus/Explicit. Wang et al. (2015) developed a parametric model for arbitrary centerline wire-rope structures and derived a series of recursive formulas for the spatial enwinding equations of wires and strands. Liang et al. (2011) calculated the equivalent elastic modulus of a wire rope in different positions based on a linear strengthening model. Bai (2011) derived a mathematical expression for the equivalent elastic modulus of a wire-rope conveyor belt based on pendency. Ma (2014) analyzed the equivalent elastic modulus of a wire-rope using static theory and validated the correctness of the analysis process by using ANSYS and by experimental measurements. Xu et al. $(2012,2015)$ proposed a method for calculating the equivalent elastic modulus of a single-fiber multidirectional winding tube based on the laminated plate theory and a theoretical estimation method for calculating the three-dimensional elastic modulus of a multifiber hybrid multidirectional winding tube considering the mixed effect.

In this study, based on the micromechanical wire-rope model, a theoretical formula for the equivalent elastic modulus of a wire rope is deduced using the differential geometry theory according to spatial characteristics of the central helical lines in the double-helix wire rope. The formula is expected to serve as a theoretical basis for dynamic analysis and optimization of mechanical systems containing wire ropes.

\section{Three-dimensional geometrical model of the wire rope}

The object of the study is a double-helix wire rope which is shown in Fig. 1. It comprises stranded ropes wound around the central strand helix according to certain rules. Each wire rope is a helical wire bundle with a high load-carrying capacity and consists of multiple wires twisted around the core wire according to a spatial spiral relationship. In this study, the spatial geometry of the wire, whose basic units are strands and ropes, is divided into the following four types: the central strand core wire, central strand side wire, lateral strand core wire, and lateral strand side wire. The central strand core wire is mostly straight or has a simple curve. The central strand side wire and the lateral strand side wire are first-degree spatial helical lines wound around the central strand core wire. The lateral strand side wire is a second-degree spatial helical line with respect to the central strand core wire. The cross section of the double-helix wire rope is shown in Fig. 1b.

To facilitate the analysis, a unit length of the twisted wire rope is intercepted. The central helical lines of the central strand core wire, central strand side wire, lateral strand core wire and the lateral strand side wire are considered separately to establish the Cartesian coordinate system, as shown in Fig. 2.

Consider a random point on the central strand side wire $P_{1}(x, y, z)$. The projection of this point on the $X O Y$ plane is $P_{1}^{\prime}$, whose coordinates are

$$
x=R_{1} \cos \theta_{1} \quad y=R_{1} \sin \theta_{1} \quad z=\frac{\theta_{1}}{2 \pi} L_{1}
$$

where $R_{1}$ is the twisted circle radius of the central strand side wire $\left(R_{1}=\left(d_{0}+d_{1}\right) / 2\right.$, where $d_{0}$ and $d_{1}$ are the radii of the central strand core wire and the central strand side wire, respectively, in millimeters), $\theta_{1}$ is the polar angle corresponding to $P_{1}$ in radians, and $L_{1}$ is the twist pitch of the central strand side wire in millimeters. 

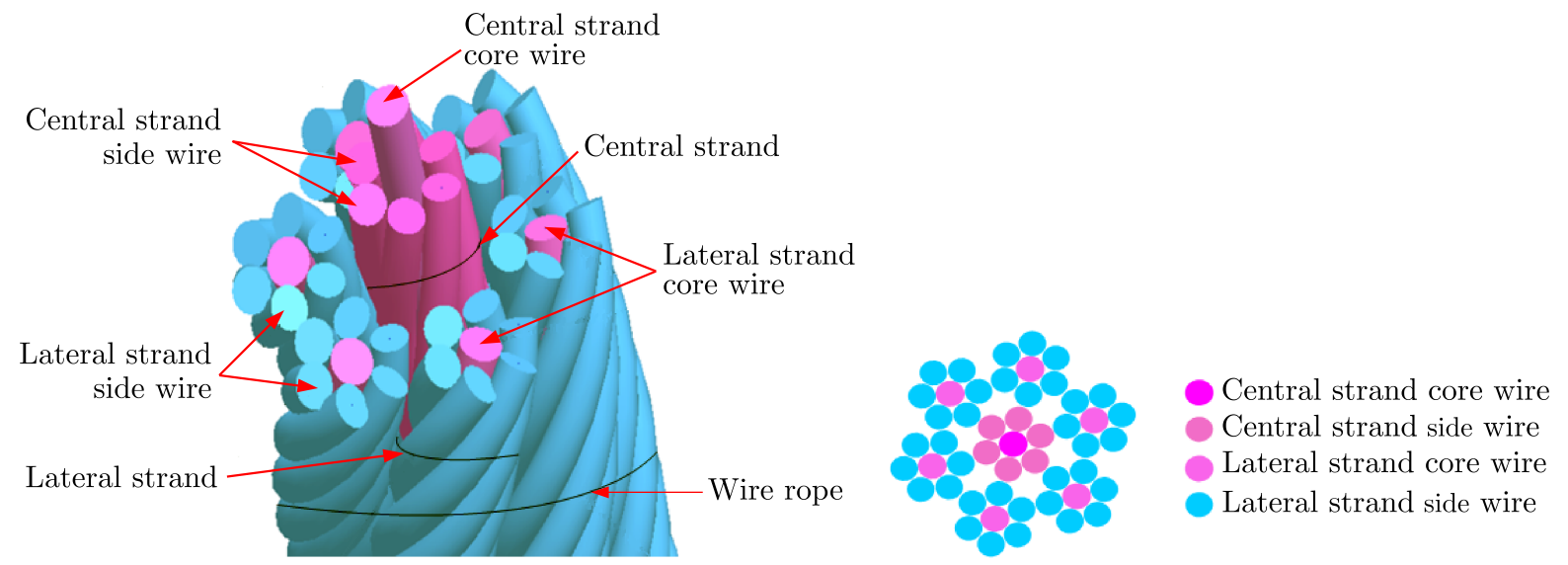

Fig. 1. Schematic diagram of the wire rope; (a) structure diagram of the wire rope, (b) cross section of the wire rope

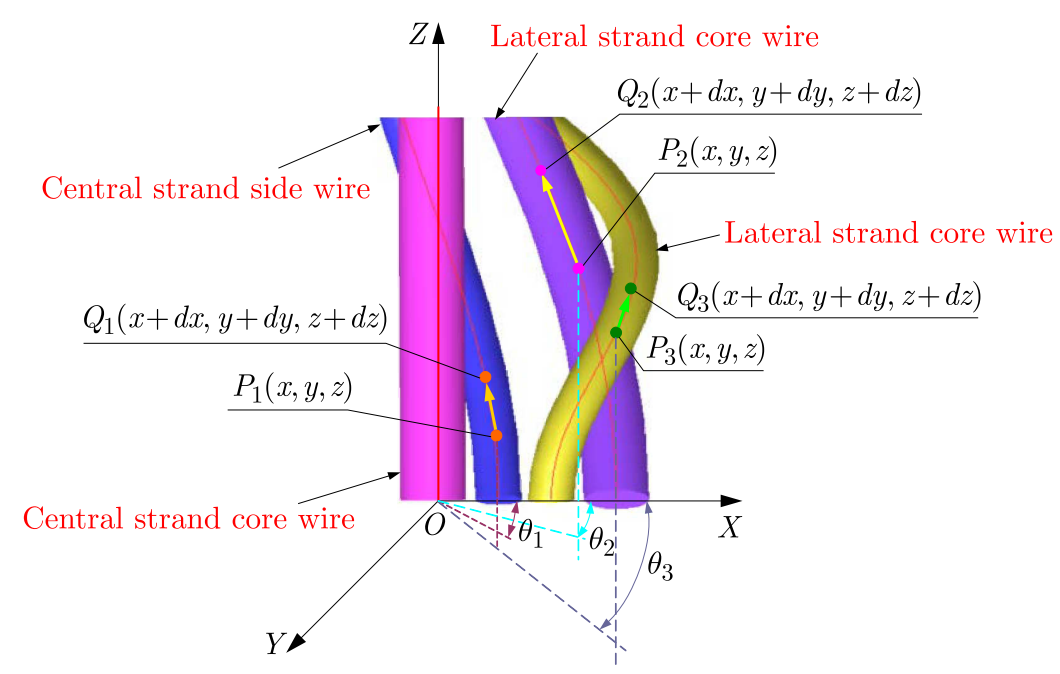

Fig. 2. Spatial coordinate system of the wire rope

Consider a small arc $P_{1} Q_{1}$ with $P_{1}$ as the starting point. Then, the coordinates of $Q_{1}$ are $(x+d x, y+d y, z+d z)$, and thus

$$
d x=-R_{1} \sin \theta_{1} d \theta_{1} \quad d y=R_{1} \cos \theta_{1} d \theta_{1} \quad d z=\frac{L_{1}}{2 \pi} d \theta_{1}
$$

The length of the small arc $P_{1} Q_{1}$ can be obtained from equations (2.1) and (2.2)

$$
d S_{1}=\sqrt{R_{1}^{2}+\left(\frac{L_{1}}{2 \pi}\right)^{2}} d \theta_{1}
$$

Thus, the length of the central strand side wire is

$$
S_{1}=\int_{0}^{2 \pi} \sqrt{R_{1}^{2}+\left(\frac{L_{1}}{2 \pi}\right)^{2}} d \theta_{1}=\sqrt{4 \pi^{2} R_{1}^{2}+L_{1}^{2}}
$$

The tangent inclination cosine of the small arc $P_{1} Q_{1}$ at $P_{1}$ on the central strand side wire is

$$
\cos \alpha_{1}=\frac{L_{1}}{\sqrt{4 \pi^{2} R_{1}^{2}+L_{1}^{2}}}=\frac{L_{1}}{S_{1}}
$$


Similarly, consider a random point on the lateral strand core wire $P_{2}(x, y, z)$. The projection of this point on the $X O Y$ plane is $P_{2}^{\prime}$, whose coordinates are

$$
x=R_{2} \cos \theta_{2} \quad y=R_{2} \sin \theta_{2} \quad z=\frac{\theta_{2}}{2 \pi} L_{2}
$$

where $R_{2}$ is the twisted circle radius of the lateral strand core wire $\left(R_{2}=\left(d_{0}+d_{2}\right) / 2+d_{1}+d_{3}\right.$, where $d_{2}$ and $d_{3}$ are the radii of the lateral strand core wire and the lateral strand side wire, respectively, in millimeters), $\theta_{2}$ is the polar angle corresponding to $P_{2}$ in radians, and $L_{2}$ is the twist pitch of the lateral strand core wire in millimeters.

Consider a small arc $P_{2} Q_{2}$ with $P_{2}$ as the starting point. Then, the coordinates of $Q_{2}$ are $(x+d x, y+d y, z+d z)$, thus

$$
d x=-R_{2} \sin \theta_{2} d \theta_{2} \quad d y=R_{2} \cos \theta_{2} d \theta_{2} \quad d z=\frac{L_{2}}{2 \pi} d \theta_{2}
$$

The length of the small arc $P_{2} Q_{2}$ can be obtained from equations (2.6) and (2.7)

$$
d S_{2}=\sqrt{R_{2}^{2}+\left(\frac{L_{2}}{2 \pi}\right)^{2}} d \theta_{2}
$$

The length of the lateral strand core wire is

$$
S_{2}=\int_{0}^{2 \pi} \sqrt{R_{2}^{2}+\left(\frac{L_{2}}{2 \pi}\right)^{2}} d \theta_{2}=\sqrt{4 \pi^{2} R_{2}^{2}+L_{2}^{2}}
$$

The tangent inclination cosine of the small arc $P_{2} Q_{2}$ at $P_{2}$ on the central strand side wire is

$$
\cos \alpha_{2}=\frac{L_{2}}{\sqrt{4 \pi^{2} R_{2}^{2}+L_{2}^{2}}}=\frac{L_{2}}{S_{2}}
$$

Consider a random point on the lateral strand side wire $P_{3}(x, y, z)$. The projection of this point on the $X O Y$ plane is $P_{3}^{\prime}$, whose coordinates are

$$
\begin{aligned}
& x=R_{2} \cos \theta_{2}-R_{3} \cos \theta_{2} \cos \theta_{3}+R_{3} \sin \beta_{2} \sin \theta_{2} \sin \theta_{3} \\
& y=R_{2} \sin \theta_{2}-R_{3} \sin \theta_{2} \cos \theta_{3}-R_{3} \sin \beta_{2} \cos \theta_{2} \sin \theta_{3} \\
& z=\frac{\theta_{2}}{2 \pi} L_{2}+R_{3} \cos \beta_{2} \sin \theta_{3}
\end{aligned}
$$

Consider a small arc $P_{3} Q_{3}$ with $P_{3}$ as the starting point. Then, the coordinates of $Q_{3}$ are $(x+d x, y+d y, z+d z)$, and thus

$$
\begin{aligned}
& d x=\left[-\frac{1}{n} R_{2} \sin \frac{\theta_{3}}{n}+\left(\frac{1}{n}+\sin \beta_{2}\right) R_{3} \sin \frac{\theta_{3}}{n} \cos \theta_{3}+\left(1+\frac{1}{n} \sin \beta_{2}\right) R_{3} \cos \frac{\theta_{3}}{n} \sin \theta_{3}\right] d \theta_{3} \\
& d y=\left[\frac{1}{n} R_{2} \cos \frac{\theta_{3}}{n}-\left(\frac{1}{n}+\sin \beta_{2}\right) R_{3} \cos \frac{\theta_{3}}{n} \cos \theta_{3}+\left(1+\frac{1}{n} \sin \beta_{2}\right) R_{3} \sin \frac{\theta_{3}}{n} \sin \theta_{3}\right] d \theta_{3} \\
& d z=\left(\frac{L_{2}}{2 \pi n}+R_{3} \cos \beta_{2} \cos \theta_{3}\right) d \theta_{3}
\end{aligned}
$$

where $R_{3}$ is the twisted circle radius of the lateral strand side wire, in millimeters $\left(R_{3}=\left(d_{0}+\right.\right.$ $\left.\left.d_{1}+d_{3}\right) / 2\right), \theta_{3}$ is the polar angle corresponding to $P_{3}$ in radians, $\beta_{2}$ is the helical ascent angle of the lateral strand side wire in radians, and $n$ is the number of twisted rounds of the lateral strand side wires about the lateral strand core wire. 
Next, set

$$
\begin{aligned}
& C_{1}=\frac{1}{n} R_{2} \quad C_{2}=\left(\frac{1}{n}+\sin \beta_{2}\right) R_{3} \quad C_{3}=\left(1+\frac{1}{n} \sin \beta_{2}\right) R_{3} \\
& C_{4}=\frac{L_{2}}{2 \pi n} \quad C_{5}=R_{3} \cos \beta_{2}
\end{aligned}
$$

The length of the small arc $P_{3} Q_{3}$ can be obtained from equations (2.11)-(2.13)

$$
d S_{3}=\sqrt{\left(C_{1}-C_{2} \cos \theta_{3}\right)^{2}+\left(C_{3} \sin \theta_{3}\right)^{2}+\left(C_{4}+C_{5} \cos \theta_{3}\right)^{2}} d \theta_{3}
$$

The length of the lateral strand side wire is

$$
S_{3}=\int_{0}^{2 \pi} \sqrt{\left(C_{1}-C_{2} \cos \theta_{3}\right)^{2}+\left(C_{3} \sin \theta_{3}\right)^{2}+\left(C_{4}+C_{5} \cos \theta_{3}\right)^{2}} d \theta_{3}
$$

The tangent inclination cosine of the small arc $P_{3} Q_{3}$ at $P_{3}$ on the central strand side wire is

$$
\cos \alpha_{3}=\frac{C_{4}+C_{5} \cos \theta_{3}}{\sqrt{\left(C_{1}-C_{2} \cos \theta_{3}\right)^{2}+\left(C_{3} \sin \theta_{3}\right)^{2}+\left(C_{4}+C_{5} \cos \theta_{3}\right)^{2}}}
$$

Combining equations (2.5), (2.10) and (2.16), the nominal area of the wire rope can be obtained

$$
A^{*}=A_{0}+6 \frac{A_{1}}{\cos \alpha_{1}}+6 \frac{A_{2}}{\cos \alpha_{2}}+36 \frac{A_{3}}{\cos \alpha_{3}}
$$

where $A_{0}, A_{1}, A_{2}$ and $A_{3}$ are the cross-sectional areas of the central strand core wire, central strand side wire, lateral strand core wire and lateral strand side wire, respectively.

\section{Calculation of the elastic modulus of the wire rope}

Because only the equivalent elastic modulus of the double-helix wire rope is calculated in this study, the following assumptions are made: (1) The cross section of the wire is perpendicular to the tangent line corresponding to the central helix. (2) Friction between the wires is negligible. (3) The wires elongate without twisting, and the elongation amount is recorded as $\Delta L$. Tension and elongation of the central strand core wire, central strand side wire, lateral strand core wire and the lateral strand side wire are deduced separately.

Ad. (1) For the central strand core wire, after an elongation of $\Delta L$ in the $Z$-axis direction, the corresponding tensile stress $\varepsilon$ and tension $T_{0}$ are respectively given as

$$
\varepsilon=E \frac{\Delta L}{L_{1}} \quad T_{0}=\varepsilon_{0} A_{0}=\frac{\pi E d_{0}^{2}}{4 L_{1}} \Delta L
$$

Because materials of the wire rope are mostly nonalloy carbon steels, the elastic modulus is assumed to be $E=183.9 \mathrm{GPa}$ (Liu, 2014).

Ad. (2) According to the helical spatial structure of the central strand side wire, the elongation can be deduced by the whole differential as follows

$$
\Delta S_{1}=\frac{4 \pi^{2} R_{1}}{S_{1}} \Delta R_{1}+\frac{L_{1}}{S_{1}} \Delta L=\frac{L_{1}}{S_{1}} \Delta L\left(1+\frac{4 \pi^{2} R_{1}}{S_{1}} \frac{\Delta R_{1}}{\Delta L}\right)
$$


According to Wang et al. (2004)

$$
1+\frac{4 \pi^{2} R_{1}}{S_{1}} \frac{\Delta R_{1}}{\Delta L} \approx 1
$$

Thus

$$
\Delta S_{1} \approx \frac{L_{1}}{S_{1}} \Delta L
$$

Based on material mechanics, the tension of a single central strand side wire in the $Z$-axis direction can be expressed as

$$
T_{1}=E \frac{\Delta S_{1}}{S_{1}} A_{1} \cos \alpha_{1}=\frac{\pi E d_{1}^{2} L_{1}^{2}}{4 S_{1}^{3}} \Delta L
$$

Ad. (3) Because the lateral strand core wire has the same spatial structure as the central strand side wire, the same method can be used to obtain expressions for the elongation and tension of the lateral strand core wire. The elongation $\Delta S_{2}$ can be expressed as

$$
\Delta S_{2} \approx \frac{L_{2}}{S_{2}} \Delta L
$$

The tension $T_{2}$ in the $Z$-axis direction is

$$
T_{2}=E \frac{\Delta S_{2}}{S_{2}} A_{2} \cos \alpha_{2}=\frac{\pi E d_{2}^{2} L_{2}^{2}}{4 S_{2}^{3}} \Delta L
$$

Ad. (4) The lateral strand side wire is the second-degree spatial helical line with respect to the

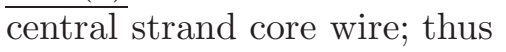

$$
\Delta S_{3}=\frac{\partial S_{3}}{\partial R_{3}}+\frac{\partial S_{3}}{\partial L_{3}} \quad T_{3}=E \frac{\Delta S_{3}}{S_{3}} A_{3} \cos \alpha_{3}=\frac{E A_{3} \cos \alpha_{3}}{S_{3}}\left(\frac{\partial S_{3}}{\partial R_{3}}+\frac{\partial S_{3}}{\partial L_{3}}\right)
$$

According to the above equations, the tension of the wire rope $T^{*}$ can be expressed as

$$
T^{*}=T_{0}+6 T_{1}+6 T_{2}+36 T_{3}
$$

The elastic modulus of the wire rope can be obtained according to equations (2.17) and (3.9)

$$
E^{*}=\frac{T^{*}}{A^{*} X}
$$

Thus

$$
E^{*}=L E \frac{\frac{d_{0}^{2}}{L_{1}}+\frac{6 d_{1}^{2} L_{1} \cos \alpha_{1}}{S_{1}^{2}}+\frac{6 d_{2}^{2} L_{2} \cos \alpha_{2}}{S_{2}^{2}}+\frac{36\left(\frac{\partial S_{3}}{\partial R_{3}}+\frac{\partial S_{3}}{\partial L_{3}}\right) d_{3}^{2} \cos \alpha_{3}}{S_{3}}}{d_{0}^{2}+\frac{6 d_{1}^{2}}{\cos \alpha_{1}}+\frac{6 d_{2}^{2}}{\cos \alpha_{2}}+\frac{36 d_{3}^{2}}{\cos \alpha_{3}}}
$$

where $X=\Delta L / L(L$ is length of the wire rope in millimeters $), S_{1}=\sqrt{4 \pi^{2} R_{1}^{2}+L_{1}^{2}}$ is length of the central strand side wire in millimeters, $S_{2}=\sqrt{4 \pi^{2} R_{2}^{2}+L_{2}^{2}}$ is length of the lateral strand side wire in millimeters, $S_{3}$ (see Eq. (2.15)) is length of the lateral strand side wire in millimeters, $\cos \alpha_{1}=L_{1} / S_{1}$ is tangent inclination cosine of the small arc on the central strand core wire, $\cos \alpha_{2}=L_{2} / S_{2}$ is tangent inclination cosine of the small arc on the lateral strand core wire, and $\cos \alpha_{3}$ (see Eq. (2.16)) is tangent inclination cosine of the small arc on the lateral strand side wire. In addition, $C_{i}(i=1, \ldots, 5)$ see Eqs. (2.13). 


\section{Case analysis and verification}

To verify the correctness of the expression for the equivalent elastic modulus predicted by the spatial distribution of the central line of the double-helix wire rope, the $6 \times 7+$ IWS wire rope is used as an example. The basic structural parameters of this rope are listed in Table 1. According to equation (3.11), the equivalent elastic modulus of the wire rope can be determined. According to the measurement method of the actual elastic modulus of a wire rope given in the national standard GB/T24191-2009, the universal tensile testing machine (Fig. 3) is used to measure the actual equivalent elastic modulus of the wire rope.

Table 1. Structural parameters of $6 \times 7+$ IWS wire rope

\begin{tabular}{|c|c|c|c|c|c|}
\hline $\begin{array}{c}\text { Wire rope } \\
\text { diameter } \\
{[\mathrm{mm}]}\end{array}$ & $\begin{array}{c}\text { Twist } \\
\text { pitch } \\
{[\mathrm{mm}]}\end{array}$ & $\begin{array}{c}\text { Number of twisted rounds } \\
\text { of lateral strand side } \\
\text { wires about lateral } \\
\text { strand core wire }\end{array}$ & \multirow{2}{*}{$\begin{array}{c}\text { Wire } \\
\text { twist } \\
\text { angle }\left[{ }^{\circ}\right]\end{array}$} & \multicolumn{2}{|c|}{$\begin{array}{c}\text { Wire-rope diameter } \\
{[\mathrm{mm}]}\end{array}$} \\
\cline { 5 - 7 } & & $\begin{array}{c}\text { central } \\
\text { strand }\end{array}$ & $\begin{array}{c}\text { lateral } \\
\text { strand }\end{array}$ \\
\hline \hline 4.5 & 36 & 3.1416 & 10.3848 & 0.6 & 0.4 \\
\hline
\end{tabular}

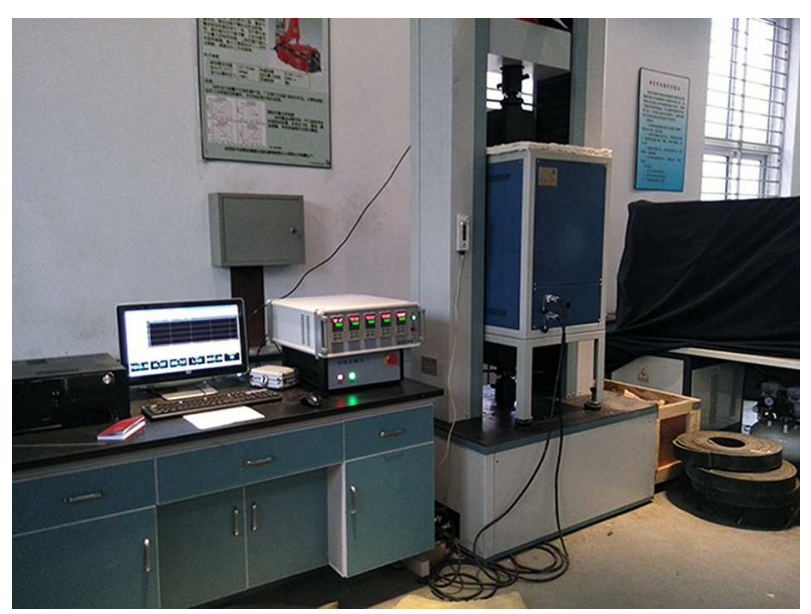

Fig. 3. Universal tensile testing machine

In the analysis, $600 \mathrm{~mm}$ of the wire rope is intercepted from selected wire-rope samples and placed at a room temperature of $18^{\circ}$ for $24 \mathrm{~h}$. Both ends of the sample are mechanically clamped in the universal tensile testing machine. Each end has clamping length of $50 \mathrm{~mm}$. The uniaxial tensile test ( $\mathrm{Wu}$ et al., 2014) is performed by clamping the wire rope through the jaws of the testing machine.

According to the requirements of the national standard GB/T24191-2009, the elastic modulus of the wire-rope sample in the fully stable state should be $10 \%-30 \%$ of the minimum breaking tension (or nominal breaking load). The loads at $10 \%$ and $30 \%$ are denoted as $F_{10 \%}$ and $F_{30 \%}$, respectively. Simultaneously, the elongations of the wire rope at $F_{10 \%}$ and $F_{30 \%}$ loads are recorded as $x_{1}$ and $x_{2}$, respectively. According to the national standard GB8918-2006, the minimum breaking tension of the $6 \times 7+$ IWS (2006) wire rope is $11.6 \mathrm{kN}$. According to the above experimental steps, the corresponding displacement-load deformation curve is obtained for the wire rope through measurement of the elastic modulus, as shown in Fig. 4.

According to the actual elastic modulus of the wire rope given in GB/T24191-2009

$$
E_{10-30}=l_{0} \frac{F_{30 \%}-F_{10 \%}}{A\left(x_{2}-x_{1}\right)}
$$




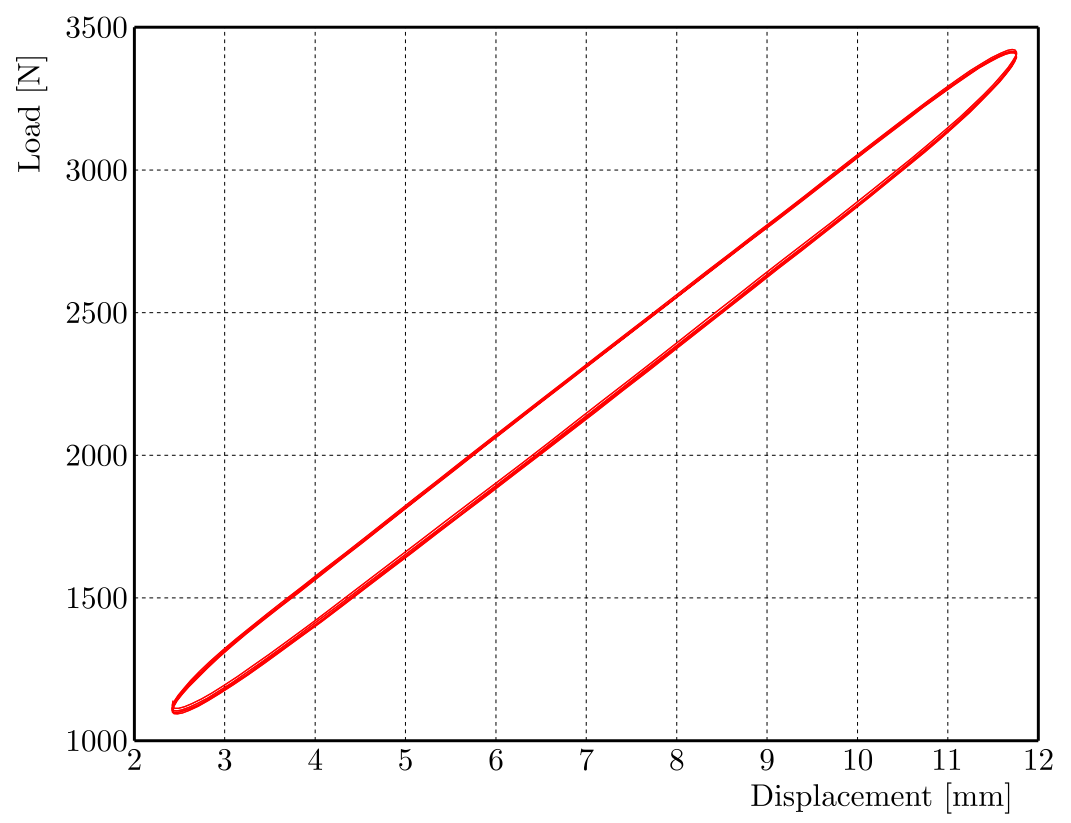

Fig. 4. Displacement-load deformation curve

where $E_{10-30}$ is the actual elastic modulus of the wire rope in gigapascals: $l_{0}$ is the initial length of the wire rope in millimeters; $F_{10 \%}$ and $F_{30 \%}$ are the loads at $10 \%$ and $30 \%$ of the minimum breaking tension (or nominal breaking load) of the wire-rope sample, respectively, in kilonewtons; $A$ is the cross-sectional area of the wire rope in millimeters (in this study $A=A^{*}$ ); and $x_{1}$ and $x_{2}$ are the elongations of the wire rope at $F_{10 \%}$ and $F_{30 \%}$ loads, respectively, in millimeters.

According to equation (4.1), the elastic modulus of the $6 \times 7+\mathrm{IWS}$ wire rope is

$$
E_{10-30}=500 \frac{3480-1160}{13.52(11.75-2.39)}=9.17 \mathrm{GPa}
$$

Compared to the theoretical value of the equivalent elastic modulus of the wire rope $E^{*}=8.75 \mathrm{GPa}$, the error of the calculated is value $4.8 \%$, which is relatively small. The results show that the value calculated using the model can be used to accurately predict the actual elastic modulus of the wire rope.

\section{Conclusion}

In this study, according to the spatial distribution characteristics of each central line of a double-helix wire rope, the theoretically predicted expression for the equivalent elastic modulus of the wire rope is obtained using the differential geometry theory. The $6 \times 7+\mathrm{IWS}$ wire rope is used as the study object, and an elastic modulus measurement experiment is performed on this rope. The correctness of the theoretical deduction of the equivalent elastic modulus is verified through the comparison and analysis of the experimental value and the theoretically predicted one.

\section{References}

1. BAI C.C., 2011, Simulation and Finite Element Analysis of the Steel-Cord Conveyer Belt on Fragmentation, Shanghai: East China University of Science and Technology, 16-18

2. Elata D., Eshkenazy R., Weiss M.P., 2004, The mechanical behavior of a wire rope with an independent wire rope core, International Journal of Solids and Structures, 41, 5-6, 1157-1172 
3. Erdönmez C., Erdem Imrak C., 2009, Modeling and numerical analysis of the wire strand, Journal of Naval Science and Engineering, 5, 1, 30-38

4. GB8918-2006, National Standards of Steel Wire Rope (Steel wire ropes for important purpose)

5. GBT228-2002, National Standards of Metallic materials -Tensile Specimens (Metallic materials Tensile testing at ambient temperature)

6. GBT24191-2009, Steel wire ropes - determination of the actual modulus of elasticity

7. Hobbs R., Nabijou S., 1995, Changes in wire curvature as a wire rope is bent over a sheave, Journal of Strain Analysis for Engineering Design, 30, 4, 271-281

8. Hu Y., Hu Z., Ma H., Yan P., Liu Y., 2016, Finite element simulation of axial elastic characteristics of wire rope with one round strand layer, IEEE International Conference on Computer Supported Cooperative Work in Design, 16-19

9. Jolicoeur C., Cardou A., 1991, A numerical comparison of current mathematical models of twisted wire cables under axisymmetric loads, Journal of Energy Resources and Technology, 113, 4, 241-249

10. Jolicoeur C., Cardou A., 1996, Semi continuous mathematical for bending of multilayered wire strands, Journal of Engineering Mechanics, 122, 7, 643-650

11. Liang Q.X., Li C., Sun J., 2011, Numerical simulation of knotted wire rope with finite element method, Journal of Taiyuan University Science and Technology, 32, 3, 232-234

12. Liu Y.H., 2014, Research on Inherent Properties and Mechanical Performances of Wire Rope, Shanxi: Taiyuan University of Technology

13. MA Q., 2014, Performance Analysis of Wire Rope Vibration Absorber, Beijing: Beijing University of Chemical Technology, 17-27

14. Ma W., Zhu Z.C., Peng Y.X., Chen G.A., 2015, Computer-aided modeling of wire ropes bent over a sheave, Advance in Engineering Software, 90, C, 11-21

15. Machida S., Durelli A.J., 1973, Response of a strand to axial and torsional displacements, Journal of Mechanical Engineering Science, 15, 4, 241-251

16. Nabijou S., Hobbs R., 1995, Relative movements within wire ropes bent over sheaves, Journal of Strain Analysis for Engineering Design, 30, 2, 155-165

17. Prawoto Y., Mazlan R.B., 2012, Wire ropes: computational, mechanical, and metallurgical properties under tension loading, Computational Materials Science, 56, 174-178

18. Sathikh S., Moorthy M., Krishnan M., 1996, Symmetric linear elastic model for helical wire strands under axisymmetric loads, Journal of Strain Analysis for Engineering Design, 31, 5, 389-399

19. Stanová E., 2013, Geometric model of the rope created of oval strands, Transport and Logistics, 1-7

20. Stanová E., Fedorko G., Fabian M., Kmet S., 2011, Computer modeling of wire stands and ropes. Part 1: Theory and computer implementation, Advance in Engineering Software, 42, 6, 305-315

21. Wang X.Y., Meng X.B., Wang J.X., Sun Y.H., Gao K., 2015, Mathematical modeling and geometric analysis for wire rope strands, Applied Mathematical Modelling, 39, 3-4, 1019-1032

22. WAng Y.J., Li Z.Q., Song X.H., 2004, Theoretical computer and analysis affecting factors on stranded wire's elastic module, Journal of Wuhan University of Technology, 26, 4, 80-82

23. Wu J., Kou Z.M., Liu Y.H., Wu G.X., 2014, Distribution of equivalent stresses and deformations for 6 strand warrington-seale rope with an independent wire rope core, Journal of China Coal Society, 39, 11, 2340-2347

24. WU W.G., CAO X., 2016, Mechanics model and its equation of wire rope based on elastic thin rod theory, International Journal of Solids and Structures, 102, 21-29 
25. Xu G.L., Ruan W.J., Wang H., Yang Q.P., 2015, Estimation of 3D effective elastic modulus for fiber multidirectional filament-wound tube, Journal of Materials Science and Engineering, 31, $1,55-59$

26. Xu G.L., Yang Q.P., Ruan W.J., Wang H., 2012, Estimation and experiment of 3D effective elastic modulus for fiber hybrid tube considering hybrid effects, Acta Materiae Compositae Sinica, 29, 4, 204-209

Manuscript received August 18, 2017; accepted for print February 6, 2018 